\title{
Successful use of emergent endovascular aneurysm repair for a ruptured hypotensive abdominal aortic aneurysm*
}

\author{
Maria Basile, Fred Fiesseler, Richard D. Shih" \\ Morristown Medical Center, Morristown, USA; \\ \#Corresponding Author: shih100@yahoo.com, mariaeve8@gmail.com, ffiesseler@yahoo.com
}

Received 12 December 2012; revised 1 April 2013; accepted 16 April 2013

Copyright (C) 2013 Maria Basile et al. This is an open access article distributed under the Creative Commons Attribution License, which permits unrestricted use, distribution, and reproduction in any medium, provided the original work is properly cited.

\begin{abstract}
Ruptured aortic aneurysm has a surgical mortality ranging from $50 \%-70 \%$, one of the highest rates of all vascular emergencies [1-5]. Less invasive approaches to repair have been developed that could potentially improve these statistics [1,5-7]. Endovascular aneurysm repair (EVAR) is a minimally invasive approach that may be an alternative to open surgical repair for select cases of ruptured aortic aneurysms [1,5]. Unfortunately, the role of EVAR in patients with acute rupture of an abdominal aneurysm is not clear, especially for patients that are hemodynamically unstable $[1,8-10]$. The literature is limited regarding use of EVAR in this population of patients. We present a case of the successful use of EVAR for an emergent repair in a hypotensive hemodynamically unstable patient with an acutely ruptured abdominal aortic aneurysm (AAA).
\end{abstract}

Keywords: Abdominal Aortic Aneurysm; Aneurysm; Endovascular Aneurysm Repair

\section{CASE REPORT}

A 66-year-old man presented to an emergency department with acute abdominal pain. The pain started abruptly and was severe in nature starting several hours before. Just prior to ED arrival, the patient had a brief syncopal episode lasting 1 - 2 minutes. He complained of nausea but denied difficulty breathing, chest pain or re-

\footnotetext{
*Grants: Emergency Medical Associates Research Foundation. No conflicts of interest.

Author Contribution Statement: RDS and FF conceived the case report and gathered the data. MB and RDS drafted the manuscript, and all authors contributed substantially to its revision. RDS takes responsibility for the paper as a whole.
}

cent illness. He had a past medical history significant for a myocardial infarction, hypercholesterolemia and hypertension. He denied smoking. His initial vital signs were temperature, $36.4^{\circ} \mathrm{C}$; heart rate, 101 beats per minute; respiratory rate, 18 per minute; and blood pressure, $97 / 56 \mathrm{mmHg}$. On physical examination, he appeared pale and in distress. His chest and cardiac examination were unremarkable. His abdomen was protuberant with marked diffuse tenderness and a pulsatile abdominal mass. A bedside ultrasound was immediately obtained that demonstrated a large AAA. Initial laboratory tests revealed a white blood cell count of $19.08 \mathrm{H} / \mathrm{nL}$, hemoglobin of $13.3 \mathrm{~g} / \mathrm{dL}$, a creatinine of $1.3 \mathrm{mg} / \mathrm{dL}$, and a troponin-I of $0.099 \mathrm{ng} / \mathrm{mL}$. The remainder of the initial laboratory tests was unremarkable. Shortly after ED arrival the patient's systolic blood pressure decreased to $60 \mathrm{mmHg}$. With intravenous fluids the systolic blood pressure rose into the 70 - $80 \mathrm{mmHg}$ range. The patient remained awake and oriented with these blood pressures.

The initial diagnosis was a hemodynamically unstable ruptured abdominal aortic aneurysm in need of acute surgical repair with a high mortality risk. The patient was considered for endovascular aneurysm repair and underwent an emergency CT scan of the abdomen and pelvis to assess eligibility for this procedure. Despite having a blood pressure that was in the $70-80 \mathrm{mmHg}$ range, the patient remained awake and alert. The patient was accompanied by the vascular surgery team to radiology while the CT scan was performed. The pre-radiology plan was if the patient decompensated during the performance of the CT scan, the vascular team would discontinue the CT scan and immediately take the patient for emergency open operative repair of the AAA.

The patient completed the CT scan without deterioration and the scan demonstrated a large infrarenal abdominal aortic aneurysm (greatest diameter $8.9 \mathrm{~cm}$ ) that had the appearance of a rupture, with a large associated 
retroperitoneal hematoma. Both the aorta and the iliac vessels demonstrated atherosclerotic disease.

The patient was immediately transferred to the operating room, where an emergent endovascular aneurysm repair procedure was begun. Femoral access was gained bilaterally. An angiogram identified the renal arteries, and the endovascular device was deployed. An abdominal aortogram demonstrated a proximal type-1 endoleak that was very slow. The proximal neck of the endovascular device was dilated to stop the endoleak, with excellent results as demonstrated via angiogram. The catheters were withdrawn and the left pulsation had diminished palpable flow. The limb was re-accessed and a retrograde angiogram demonstrated another endoleak, as well as a significantly tortuous right common iliac. Additional measures were taken to fix the endoleak via angioplasty. A completion angiogram demonstrated an excellent result with brisk flow into the hypogastrics and no endoleak. The patient was out of bed on post-operative day 1 and ambulatory on day 2. Follow up CT scan of post-operative day 3 demonstrated the aortic aneurysm sac was unchanged in size and appearance, with no endoleak detected. There was an interval decrease of the retroperitoneal hematoma. The patient remained free of pain and recovered uneventfully. He was discharged from the hospital on post-operative day 3. At three month follow-up the patient continues to do well without the development of additional complications.

\section{DISCUSSION}

Abdominal aortic aneurysms (AAA) are associated with a mortality rate of less than 5 percent when repaired electively before rupture [1,5]. However, emergent repair of a ruptured AAA is associated with mortality rates greater than $50 \%$ [1,5]. Ruptured AAA carries one of the highest surgical mortalities of all vascular emergency medical conditions [1-5]. Endovascular aneurysm repair of ruptured AAAs represents a viable alternative to traditional open repair that may decrease this high mortality rate $[1,5]$.

Endovascular aneurysm repair (EVAR) is a minimally invasive approach that may be an alternative to open surgical repair for EVAR-suitable ruptured aortic aneurysms in select patient populations. Endovascular aneurysm repair is akin to operating on an aneurysm from the inside of the blood vessel, taking away the need for open surgery. A common approach is a small incision made in the femoral artery that provides a pathway through which a catheter is threaded. Once at the site of the weakened vessel wall, a balloon is inflated to hold open the blood vessel lumen, and a mesh stent is placed as scaffolding to strengthen the wall and prevent rupture.

The use of EVAR for abdominal aortic aneurysm offers the potential advantage of avoiding the complica- tions associated with a large abdominal surgical incision, avoiding the stress on organs and tissues induced by the need to cross clamp the aorta, decreasing bleeding and inflammatory response from surgical tissue dissection, reducing risk of multiorgan failure, lessening the requirement for deep anesthesia, and providing a repair mechanism for patients who are too high risk for open repair surgical procedures due to cardiac risk factors or other comorbidity [1,5-9,11-13]. Endovascular aneurysm repair also offers the benefit of shorter hospital stays, reduced recovery time, less post-procedural pain, and avoids the complication of adhesive intestinal obstruction when compared to open repair [1,4-9]. However, a negative aspect for EVAR is the possibility of graft movement after treatment that could allow reinstatement of the aneurysm $[1,4$, 10,13]. In addition, EVAR is associated with the development of endoleaks, which require repair. Both of these potential complications require lifelong surveillance $[1,4$, 6-7,10,13].

In patients with AAA that are not ruptured, EVAR does not appear to offer a mortality benefit. In patients with ruptured AAA, EVAR appears to offer a decreased operative mortality [1,5-10]. Unfortunately, this information is derived from retrospective studies or case series with little randomized prospective data available, and the available studies may not adequately adjust for selection bias $[4,6-10,14,15]$. There is a tendency for more severely unstable patients requiring a faster treatment decision to undergo traditional open repair, and because circulatory shock is a significant prognostic factor, this has been suggested to falsely improve the favorable EVAR results [1,2,4,6-10,16-18]. However, in the available reported literature, patients who undergo EVAR for ruptured AAA tend to have greater co-morbidities and risk factors than those who are given open repair, and have a greater time delay to treatment due to CT scanning to confirm rupture $[1,10,11,15,18]$. Unfortunately, even less data is available regarding the subset of patients, similar to our presented case, involving ruptured and hemodynamically unstable AAA $[10,12]$. Few cases demonstrate the successful use of EVAR in hemodynamically unstable AAA. In cases like ours where patients represent high surgical mortality risk, EVAR may be a useful alternative [3-10,11-13,16,17,19,20]. We present our case as a piece of the growing evidence that EVAR may be useful in patients with a ruptured AAA that are also hemodynamically unstable.

\section{CONCLUSION}

Endovascular aneurysm repair is being increasingly employed in place of open surgical repair in elective abdominal aortic aneurysm repair procedures. Its use in emergent repair of hemodynamically unstable ruptured abdominal aortic aneurysm is controversial and not as 
well documented as EVAR in stable patients [10,11-13, 19,20]. Our case demonstrates a successful example of EVAR utilized in a hemodynamically unstable patient with a ruptured abdominal aortic aneurysm in a patient with a high pre-operative surgical mortality risk. The use of EVAR in this case resulted in minimal hospital recovery time and good outcome. We provide new evidence and support for the growing interest in the use of EVAR for hemodynamically unstable patients.

\section{REFERENCES}

[1] Moll, F.L., et al. (2011) Management of abdominal aortic aneurysms clinical practice guidelines of the european society for vascular surgery. European Journal of Vascular and Endovascular Surgery, 41, S1-S58. doi:10.1016/j.ejvs.2010.09.011

[2] Bonardelli, S., et al. (2011) Open surgery in endovascular aneurysm repair era: Simplified classification in two risk groups owing to factors affecting mortality in 137 ruptured abdominal aortic aneurysms (RAAAs). Updates in Surgery, 63, 39-44. doi:10.1007/s13304-011-0053-Z

[3] Katz, D.J., Stanley, J.C. and Zelenock, G.B. (1994) Operative mortality rates for intact and ruptured abdominal aortic aneurysms in Michigan: An eleven-year statewide experience. Journal of Vascular Surgery, 19, 804-817. doi:10.1016/S0741-5214(94)70005-2

[4] Egorova, N., et al. (2008) National outcomes for the treatment of ruptured abdominal aortic aneurysm: Comparison of open versus endovascular repairs. Journal of Vascular Surgery, 48, 1092-100. doi:10.1016/j.jvs.2008.06.036

[5] Veith, F.J., et al. (2003) Treatment of ruptured abdominal aneurysms with stent grafts: A new gold standard? Seminars in Vascular Surgery, 16, 171-175. doi:10.1016/S0895-7967(03)00003-6

[6] Larzon, T., Lindgren, R. and Norgren, L. (2005) Endovascular treatment of ruptured abdominal aortic aneurysms: A shift of the paradigm? Journal of Endovascular Therapy, 12, 548-555. doi:10.1583/04-1469.1

[7] Lyons, O.T.A., et al. (2010) Emergency endovascular aneurysm repair for ruptured abdominal aortic aneurysm: The way forward? Vascular, 18, 130-135. doi:10.2310/6670.2010.00033

[8] Karkos, C.D., et al. (2009) Mortality after endovascular repair of ruptured abdominal aortic aneurysms: A systematic review and meta-analysis. Archives of Surgery, 144, 770-778. doi:10.1001/archsurg.2009.132

[9] Azizzadeh, A., et al. (2008) Endovascular repair of rup- tured abdominal aortic aneurysms: Systematic literature review. Vascular, 16, 219-224. doi:10.2310/6670.2008.00039

[10] Veith, F.J., et al. (2010) Is a randomized trial necessary to determine whether endovascular repair is the preferred management strategy in patients with ruptured abdominal aortic aneurysms? Journal of Vascular Surgery, 52, 10871093. doi:10.1016/j.jvs.2010.05.142

[11] Gerassimidis, T.S., et al. (2005) Endovascular management of ruptured abdominal aortic aneurysms: 6-year experience from a Greek center. Journal of Vascular Surgery, 42, 615-623. doi:10.1016/j.jvs.2005.05.061

[12] Franks, S., et al. (2006) Endovascular treatment of ruptured and symptomatic abdominal aortic aneurysms. European Journal of Vascular and Endovascular Surgery, 31, 345-350. doi:10.1016/j.ejvs.2005.08.037

[13] Hinchliffe, R.J., Braithwaite, B.D. and Hopkinson, B.R. (2003) The endovascular management of ruptured abdominal aortic aneurysms. European Journal of Vascular and Endovascular Surgery, 25, 191-201. doi:10.1053/ejvs.2002.1846

[14] Hinchliffe, R.J., et al. (2006) A randomised trial of endovascular and open surgery for ruptured abdominal aortic aneurysm-Results of a pilot study and lessons learned for future studies. European Journal of Vascular and Endovascular Surgery, 32, 506-515. doi:10.1016/j.ejvs.2006.05.016

[15] Hinchliffe, R.J., et al. (2009) Endovascular repair of ruptured abdominal aortic aneurysm: A strategy in need of definitive evidence. Journal of Vascular Surgery, 49, 1077 1080. doi:10.1016/j.jvs.2009.02.005

[16] Veith, F.J., et al. (2009) Collected world and single center experience with endovascular treatment of ruptured abdominal aortic aneurysms. Annals of Surgery, 250, 1-7. doi:10.1097/SLA.0b013e3181bdd7f5

[17] Vaddineni, S.K., et al. (2005) Ruptured abdominal aortic aneurysm: A retrospective assessment of open versus endovascular repair. Annals of Vascular Surgery, 19, 782786. doi:10.1007/s10016-005-7975-1

[18] Hinchliffe, R.J. and Braithwaite, B.D. (2007) Ruptured abdominal aortic aneurysm: Endovascular repair does not confer any long-term survival advantage over open repair. Vascular, 15, 191-196. doi:10.2310/6670.2007.00045

[19] Van Herzeele, I., et al. (2003) Endovascular repair of aortic rupture. European Journal of Vascular and Endovascular Surgery, 26, 311-316. doi:10.1053/ejvs.2002.2007

[20] Mehta, M., et al. (2006) Establishing a protocol for endovascular treatment of ruptured abdominal aortic aneurysms: Outcomes of a prospective analysis. Journal of Vascular Surgery, 44, 1-8. doi:10.1016/j.jvs.2006.02.057 